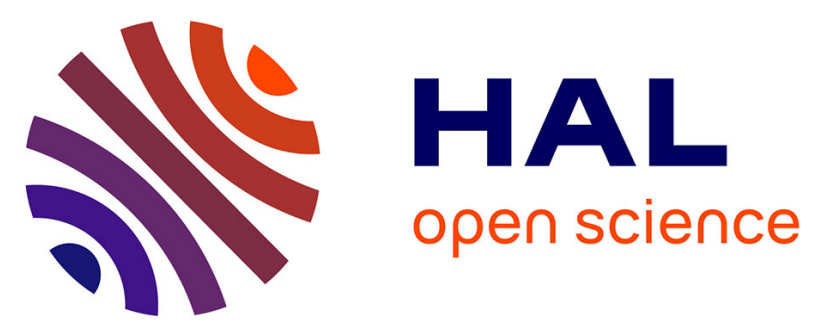

\title{
TexiCare: An innovative embedded device for pressure ulcer prevention. Preliminary results with a paraplegic volunteer.
}

Olivier Chenu, Nicolas Vuillerme, Marek Bucki, B. Diot, Francis Cannard, Yohan Payan

\section{To cite this version:}

Olivier Chenu, Nicolas Vuillerme, Marek Bucki, B. Diot, Francis Cannard, et al.. TexiCare: An innovative embedded device for pressure ulcer prevention. Preliminary results with a paraplegic volunteer.. Journal of Tissue Viability, 2013, 22, pp.83-90. 10.1016/j.jtv.2013.05.002 hal-00844476

\section{HAL Id: hal-00844476 https://hal.science/hal-00844476}

Submitted on 15 Jul 2013

HAL is a multi-disciplinary open access archive for the deposit and dissemination of scientific research documents, whether they are published or not. The documents may come from teaching and research institutions in France or abroad, or from public or private research centers.
L'archive ouverte pluridisciplinaire $\mathbf{H A L}$, est destinée au dépôt et à la diffusion de documents scientifiques de niveau recherche, publiés ou non, émanant des établissements d'enseignement et de recherche français ou étrangers, des laboratoires publics ou privés. 
TexiCare: An innovative embedded device for pressure ulcer prevention. Preliminary results with a paraplegic volunteer.

Olivier Chenu ${ }^{1}$, Nicolas Vuillerme ${ }^{2,3}$, Marek Bucki ${ }^{1}$, Bruno Diot ${ }^{4}$, Francis Cannard ${ }^{1}$ and Yohan Payan ${ }^{5}$

(1) TexiSense Company, Montceau-les-Mines, F-71300, France

(2) AGIM FRE 3405 CNRS / UJF Grenoble 1 / UPMF / EPHE, Grenoble, F-38041, France

(3) Institut Universitaire de France

(4) IDS Company, Montceau-les-Mines, F-71300, France

(5) UJF-Grenoble 1 / CNRS / TIMC-IMAG UMR 5525, Grenoble, F-38041, France

Corresponding author: Yohan Payan, PhD; phone: (+33)4.56.52.00.01; fax:

(+33)4.56.52.00.55; e-mail: yohan.payan@ imag.fr (publishable)

Source of supports: equipment provided by TexiSense SA

Word count: 2559 (abstract: 196)

Number of figures: 9

Number of tables: 0 


\title{
TexiCare: An innovative embedded device for pressure ulcer prevention. Preliminary results with a paraplegic volunteer.
}

\begin{abstract}
and key terms
Abstract - This paper introduces the recently developed TexiCare device that aims at preventing pressure ulcers for people with spinal cord injury. This embedded device is aimed to be mounted on the person's wheelchair. Its sensor is $100 \%$ textile and allows the measurement of pressures at the interface between the cushion and the buttocks. It is comfortable, washable and low cost. It is connected to a cigarette-box sized unit that (i) measures the pressures in real time, (ii) estimates the risk for internal over-strains, and (iii) alerts the wheelchair user whenever necessary. The alert method has been defined as a result of a utility / usability / acceptability study conducted with representative end users. It is based on a tactile-visual feedback (via a watch or a smartphone for example): the tactile modality is used to discreetly alarm the person while the visual modality conveys an informative message. In order to evaluate the usability of the TexiCare device, a paraplegic volunteer equipped his wheelchair at home during a six months period. Interestingly, the first results revealed bad habits such as an inadequate posture when watching TV, rare relief maneuvers, and the occurrence of abnormal high pressures.
\end{abstract}

Keywords—-pressure ulcer, deep tissue injury, prevention, smart textile. 


\section{TexiCare: An innovative embedded device for pressure ulcer prevention. Preliminary results with a paraplegic volunteer.}

\section{Introduction}

Pressure ulcers represent one of the most common, disruptive, disabling and even life threatening conditions affecting people with spinal cord injury (SCI) and other wheelchair users [1]. Whereas it can take only few minutes for a pressure ulcer to develop, complete healing can need many months of hospital time, requiring difficult and expensive medical and/or surgical treatments. Accordingly, clinicians fully agree that it is far better to prevent pressure ulcers than to treat them [2]. Unfortunately, current available preventive techniques, mainly based on the improvement of the skin/support interface and on postural and behavioral education are not efficient enough: the prevalence of pressure ulcers in people with SCI still remains very high [1], such that development and validation of innovative technological devices to prevent pressure ulcers is strongly needed.

Our group is working on pressure ulcer prevention since almost ten years. Based on the concept of "sensory substitution" [3], we have proposed the principle of supplying the wheelchair user with supplementary sensory information regarding the detection and the localization of excessive dangerous seated buttock pressures [4-6]. Indeed, contrary to healthy individuals, i.e. with intact sensory capacities, people with SCI do not get the feedback arising from the buttocks informing them of a localized excess of pressure at the skin/seat interface and the necessity to make adaptive postural correction to prevent pressure ulcers.

Our experience in this domain and the results of a participatory user-centered design study conducted with representative end users allowed us to define the main bottlenecks that need 
to be solved for the design and for a daily-life use of a personalized monitoring device embedded on the wheelchair of paraplegic persons [7]:

- $\quad$ Each patient should be able to buy and keep his own device at home. Such a device should therefore be designed with this in mind, by keeping the manufacturing costs low. Note that nothing in such a perspective is proposed on the market today, and that the companies that sell pressure map sensors alone for wheelchair cushions propose very high device costs (around $15 \mathrm{k} \$$ each) that cannot be afforded by patients.

- $\quad$ The pressure map should be comfortable and washable.

- In case of risk of pressure ulcer, the alert sent to the person should remain discrete but easily perceptible.

- $\quad$ The device has to be personalized. Indeed, it is known that the risks depends on the morphology of the person as well as on his/her postural behavior and habits [8].

This paper introduces the very recently developed TexiCare device. It is made of three components: (1) a fully wireless, customizable and washable map made of a textile able to measure pressures, (2) an electronic central unit, linked to the pressure map and wirelessly connected to (3) a tactile-visual alert device. In terms of emerging technologies, we think the $100 \%$ textile pressure map is definitively the main result of our novel research. This is completed with an embedded electronic central unit that analyzes the pressure patterns and estimates the risks, taking into account the morphology of the person. As a first step, the feasibility of daily usage of the TexiCare device had to be verified. In that aim, a single paraplegic person volunteered to be equipped at his home for a period of six months by a light version of the device. Indeed, at this step we did not want to evaluate the efficiency of the device yet, but rather its acceptability during routine usage. In consequence, the alert 
device was not installed in that light version of the TexiCare device and the algorithm that estimates the risks for pressure ulcers was not used. Pressure data were collected, analyzed and a posteriori correlated with the person's activities. This was done by installing video cameras in the person's house allowing an a posteriori synchronization between the pressure recordings and the daily living activities of the person.

\section{Material and methods}

\section{a) Material: the TexiCare device}

\section{i. $\quad$ General description}

Fig. 1 illustrates the general principle of the TexiCare device.

The textile map is put onto the chair's seat area, usually all around the cushion (Fig. 2a). An electronic central unit (size $12 \times 8 \times 2 \mathrm{~cm}$, Fig. 2b) is connected to this textile map, allowing a continuous and real-time acquisition of the pressure applied on the seat/skin interface. As it was originally suggested by previous works $[9,10]$, this central unit can estimate the internal stresses and strains due to the external surface pressures. It is indeed known that pressure ulcers mostly result from deep tissue injuries arising at the soft tissue / bone interfaces [11]. Assuming that we would be able to quantify the subject-specific pressure ulcer risk (in terms of maximal stresses or strains that can be accepted by the tissues), the central unit can estimate which postural modification would reduce the stress/strain concentration [4-6]. The user is then (i) alerted via wrist vibratory stimulation provided by a custom watch (Fig. 1, left) and/or (ii) informed of the localization of the excessive buttock pressure via a visual display (e.g., Smartphone or tablet, Fig. 1, right). 


\section{ii. A textile able to measure the pressures}

As explained above, the pressure sensors embedded in the wheelchair have to be comfortable since they sit on the cushion. Moreover, contrary to the sensors provided by the available commercial products such as XSensor, Vista Medical or Tekscan (dedicated to reeducation centers), our sensors rely on a low cost technology. Our patented textile, able to measure the pressures applied to it, is made of three layers (Fig. 3). Two outer layers form a matrix that defines the spatial resolution of the sensor. The middle layer provides a piezo-resistive effect: any normal forces exerted onto the textile fibers change the electrical resistance of the material. The innovative part of this device is that this is the textile itself that (1) conducts the current for the upper and lower layers and (2) has a piezo-resistive effect for the intermediary layer. Indeed, the outer layers are made of nylon fibers coated with silver; they can therefore conduct current. The fibers of the intermediary layer are coated with polymers having a piezo-resistive effect. Once the three layers are stacked together, the piezo elements of the intermediary layer are sandwiched between lines of electrical buses (Fig. 3). For each row of the lower matrix, the electronic system "scans" each column of the upper matrix for the resistance value of the piezo fiber sensors. The change in pressure resulting in a change in resistance values is converted into a voltage that is measured by the central unit.

The textile matrices thus generated can easily be integrated into clothing or placed on any surface requiring pressure, strain or tension measurements. Due to its inherent flexibility (Fig. 2c), the textile sensor can perfectly match any anatomical area where loading is encountered, such as the buttock area for a seated individual. Moreover, the fabric is low cost and the textile part can easily be unplugged from the rest of the device, making it washable and semi-disposable. Some preliminary laundering tests (in washing machine) have been held and yield the conclusion that the map can be washed for at least two or 
three times before changes in the properties of the piezo-resistive layers necessitate replacement of the textile component.

This technology makes it possible to create a textile with the desired number of pressuresensing areas (from a few up to thousands of spots). In the case of the TexiCare fabric, a device with 1024 piezo-resistive sensors arranged in a 32 x 32 matrix was proposed (Fig. 4). Each sensor is about $1 \mathrm{~cm}^{2}$ and the total size corresponds to the wheelchair sitting area. The device is able to acquire one pressure map every 2 seconds, which is probably sufficient for pressure ulcer prevention. A calibration process has been defined to verify that the pressure values reported by the TexiCare map (ranged between 0 and $1000 \mathrm{mmHg}$ which corresponds to forces between 0 and $13.35 \mathrm{~N}$ ) match the measurements provided by a manometer (Sauter FH 50, range 0-50N, precision 0.01N).

\section{b) Method: Monitoring at home}

A volunteer paraplegic person (male, 70 years old, $70 \mathrm{~kg}$, level of injury : T4, six pressure ulcers developed since his injury in 1985 , none since this experiment) was equipped at home with the TexiCare device during a six month period in the year 2009 (Fig. 5). At the time of this experiment, this person had mainly sedentary activities and used a traditional manual wheelchair with a gel cushion (Jay® Active). The textile was put on top of the cushion. The central unit was equipped with a Flash SD card that stored the pressure data.

The study was respectful of the French laws handling the treatment of private health data (March 4 ${ }^{\text {th }}, 2002$ and August 13 ${ }^{\text {th }}, 2004$ laws).

In order to a posteriori correlate the recorded pressure patterns with the activity of the paraplegic volunteer, an agreement was obtained to install video cameras inside the house. The goal was, in case of specific "events" detected by the pressure map, to be able to know 
afterwards what the person was doing during these events. The agreement was obtained by the ethics committee of the Grenoble Hospital (Service d'Hospitalisation à Domicile HAD, Prof. A. Franco), under the condition that only the volunteer had access to the films captured by the cameras. If the researchers wanted to know more about a specific event, they provided the time code of the event and asked the person to look at the correspondence in the camera recordings. Then, the volunteer decided to mention (or not to mention) what he was actually doing during that event. All the data were managed by IDS Company that has signed an agreement with the French Ministry of Health in terms of security/anonymity of the data.

\section{Results}

The 6 months of recordings corresponded to 1.25 Gbytes of data. General trends were observed (Fig. 6) such as regular (sleep, naps, awake hours) and non-regular events (changes in the time line of the day, transfers, relief maneuvers). It was thus clearly observed that the volunteer had a very regular activity during the day, with some changes occurring during the week-ends.

Another clear result concerned the fact that high pressures around the ischia appeared often at the end of the day, after many hours spent in the wheelchair (see Fig. 7). This is probably due to the fact that the person uses a cushion which includes a gel that tends to move to the periphery of the cushion after being pressed by the buttock area. This observation was

provided to the volunteer during the study. Thanks to this, he thus adopted a new strategy consisting in pushing the gel back to the center of the cushion after each transfer, which was much more efficient in terms of pressure smoothing. 
Another observation concerned the posture of the person when watching TV. It appeared that the volunteer frequently had asymmetrical sitting postures during that activity. A posteriori, looking at the video provided the explanation: the person was doing crosswords with notebooks laid on a table positioned laterally to the wheelchair. Again, such observations allowed the volunteer to change his postural behavior for such a task, allowing him to decrease the risk of pressure ulcer formation in such a situation.

Finally, our last result concerns the relief maneuvers of the subject. Generally, relief maneuvers are defined as events of very low external pressures (nearly zero). More logically, recent studies suggested defining them as very low internal tissue stresses [10]. In our case, the subject was not able to fully decrease his buttocks pressures, but his attempts were clearly visible on the data. In consequence, for our study, it was decided to label an event as a "relief maneuver" if the total load recorded on the map was reduced by $50 \%$. The total load is equivalent to the weight supported by the cushion; it was defined as the sum of the external forces measured by each textile sensor (the force on a given sensor is the measured pressure value multiplied by the sensor area). The triangles in Fig. 6 show the automatic detections of these events. During the period of this study, the subject was seated in his wheelchair on average 9.8 hours per day, but he did only 4.3 relief maneuvers per day (s.d. 2.7). These lift-offs lasted for 7.7 seconds each (s.d. 5.4). This means that the paraplegic volunteer relieved his weight only 0.44 times per hour, which is very little compared to what is usually recommended by clinicians (generally one maneuver per hour). It even happened that no relief maneuvers at all were done for more than ten consecutive hours during which the subject remained seated (Fig. 6, Friday). 


\section{Discussion and perspectives}

This paper has introduced an embedded, low-cost and ergonomic pressure ulcer prevention device. The principle is to record and analyze in real-time the buttock pressure map. A first prototype was tested by a paraplegic volunteer and allowed to observe a number of interesting data, like the number and duration of reliefs, the arising of abnormal high pressures and bad postural behaviors. Of course, these results should be considered as very preliminary, with a single test case.

Another innovation of the TexiCare device (not studied here) is its ability to include a module that takes into account the specific anatomy of the person's buttock area. This module is made of a 3D Finite Element model of the soft tissues that can, in real-time, estimate the internal stresses and strains resulting from a given pressure map (Fig. 8, see [12] for details concerning the real-time computation). It is indeed known that the pressure cartography measured at the interface between the skin tissues and the cushion is not sufficient to inform about risks, since the high stresses and strains at the origin of pressure ulcers are usually located inside the tissues, at the interface with the prominent bony structures such as the ischium or the trochanter bone [11]. It was unfortunately not possible to use that Finite Element module with the paraplegic volunteer since at the time this study was conducted (1) no imaging data (such as MRI, CT, US or surface scans) were available for that person and (2) we were still lacking accurate information concerning the maximal stresses and strains that can be supported by the buttock soft tissues.

As a continuation to this preliminary study, we are now starting a much larger clinical study including 90 patients from three French Hospitals. The TexiCare device used for this clinical study will include person-specific Finite Element models of the buttock soft/hard tissues since MRI data will be collected for some patients (for other patients, the Mesh- 
Match-and-Repair algorithm, as proposed by our group [13] will be used to generate subject-specific Finite Element model : see [14] for details). To estimate the in vivo patientspecific constitutive behavior of the skin tissues, the LASTIC device developed by our group will be used [15]. Concerning the estimation of the maximal stresses and/or strains that can be supported by soft tissues (Fig. 9), we will rely on the recent results provided by [16], proposing two strain thresholds that should be monitored in order to avoid any deep tissue injury. These thresholds follow many other works that tried to provide quantitative data on the risks for pressure ulcers [10, 17-19].

We believe that the TexiCare device should be useful for paraplegic people and may help them to monitor their interface pressures and prevent pressure ulcers. 


\section{References}

1. Chen Y, DeVivo M, Jackson A. Pressure ulcer prevalence in people with spinal cord injury: age-period-duration effects. Arch. of phys. Med. and rehabil. $2005 ; 86: 1208-1213$.

2. Miot R. Analyse coût-efficacité de la prévention et du traitement des escarres. L'escarre $1999 ; 4: 38-39$.

3. Bach-y Rita P, Collins CC, Saunders FA, White B, Scadden L. Vision substitution by tactile image projection. Nature $1969 ; 221: 963-964$.

4. Chenu O. Conception et validation d'un dispositif de suppléance perceptive dédié à la prévention des escarres. Grenoble: Université Joseph Fourier 2009 ; 194 pp.

5. Chenu O, Payan Y, Hlavackova P et al. Pressure sores prevention for paraplegic people: effects of visual, auditive and tactile supplementations on overpressures distribution in seated posture. Appl. Bionics and Biomech. 2012 ; 9:61-67.

6. Chenu O, Vuillerme N, Demongeot J, Payan Y. A wireless lingual feedback device to reduce overpressures in seated posture: A feasibility study. PLoS ONE 2009 ; 4 : e7550.

7. Chenu O, Payan Y, Hlavacková P, Bucki M, Diot B, Cannard F, Demongeot J, Vuillerme N. System for pressure sore prevention: a user-centered design. 14th Annual European Pressure Ulcer Advisory Panel Meeting (EPUAP) "Pressure Ulcer Research Achievements Translated to Clinical Guidelines”, Oporto (Portugal), August 31 September 2, 2011.

8. Byrne DW, Salzberg CA. Major risk factors for pressure ulcers in the spinal cord disabled : a literature review. Paraplegia $1996 ; 34$ : 255-263. 
9. Agam L, Gefen A. Toward real-time detection of deep tissue injury risk in wheelchair users using Hertz contact theory. J. Rehabi.1 Res. Dev. 2008 ; 45 : 537-550.

10. Linder-Ganz E, Yarnitzky G, Yizhar Z, Siev-Ner I, Gefen A. Real-time finite element monitoring of sub-dermal tissue stresses in individuals with spinal cord injury: toward prevention of pressure ulcers. Ann. Biomed. Eng. 2009 ; 37 : 387-400.

11. Berlowitz DR, Brienza DM. Are all pressure ulcers the result of deep tissue injury? A review of the literature. Ostomy Wound Manag $2007 ; 53: 34$.

12. Bucki M, Luboz V, Lobos C, Vuillerme N, Cannard F, Diot B, Payan Y. Patientspecific finite element model of the buttocks for pressure ulcer prevention - linear versus non-linear modelling. Computer Methods in Biomechanics and Biomedical Engineering. $2012 ; 15$ : 38-40.

13. Bucki M., Lobos C. \& Payan Y. (2010). A Fast and Robust Patient Specific Finite Element Mesh Registration Technique: Application to 60 Clinical Cases. Medical Image Analysis, Vol. 14, pp. 303-317.

14. Bucki M, Payan Y, Cannard F, Diot B, Vuillerme N. Multi-Modal Framework for Subject-Specific Finite Element Model Generation aimed at Pressure Ulcer Prevention. Proc. of the "38ème Congrès de la Société de Biomécanique", Comput. Methods in Biomech. and Biomed. Eng. 2013.

15. Schiavone P, Promayon E, Payan Y. LASTIC: a Light Aspiration device for in vivo Soft TIssue Characterization. Lecture Notes in Computer Science. 2010 ; 5958 : 1-10 
16. Loerakker S, Manders E, Strijkers GJ, Nicolay K, Baaijens FPT, Bader DL, Oomens CWJ. The effects of deformation, ischaemia and reperfusion on the development of muscle damage during prolonged loading. J. Appl. Phys. 2011 ; 111(4) : 1168-1177.

17. Gefen A, van Nierop B, Bader D, Oomens C. Strain-time cell-death threshold for skeletal muscle in a tissue-engineered model system for deep tissue injury. J. of Biomech. $2008 ; 41: 2003-2012$.

18. Sopher R, Nixon J, Gorecki C, Gefen A. Effects of Intramuscular Fat Infiltration, Scarring, and Spasticity on the Risk for Sitting-Acquired Deep Tissue Injury in Spinal Cord Injury Patients. J. Biomech. Eng. 2011 ; 133(2) : 021011.

19. Slomka N, Gefen A. Relationship between strain levels and permeability of the plasma membrane in statically stretched myoblasts. Ann Biomed Eng. 2012 Mar;40(3):606-18. 


\section{Illustration legends}

1. Principle of the TexiCare device: a dangerous situation triggers a wireless alert to the user by the mean of a watch or more sophisticated devices.

2. (a) TexiCare map around the cushion of a wheelchair ; (b) The central unit connected to the map is approximately a cigarette box sized ; (c) The TexiCare textile is thin, comfortable and washable.

3. The three layers that constitute the TexiCare textile sensors.

4. The 32x32 TexiCare sensors textile matrix ; (a) data collected from the device that reflect the pressures applied at the textile surface (b).

5. The paraplegic volunteer at home. His wheelchair is equipped with the TexiCare device.

6. Total load recorded during a week (top: Monday; down: Sunday). The periods with no data indicate an absence of the user on the wheelchair; the triangles are automatic detections of the relief maneuvers.

7. High pressures recorded during a (Dark grey parts occurring Thursday and Friday afternoon might be dangerous).

8. Person-specific Finite Element model: internal strains are computed as a result of pressure exerted on the skin surface .

9. Accumulation of strain: a strain level applied during a short period (a) can become dangerous if it is maintained (b). 


\section{Acknowledgments}

This work was carried out with the financial support of IDS Company, Carnot LSI Institute and the French national research project ANR/TecSan 2010 IDSocks. The authors wish to thank Alain Franco and Lydie Nicolas from the Grenoble Hospital and the HAD Service. We are also grateful to Jean-Paul for his kindness and availability. 


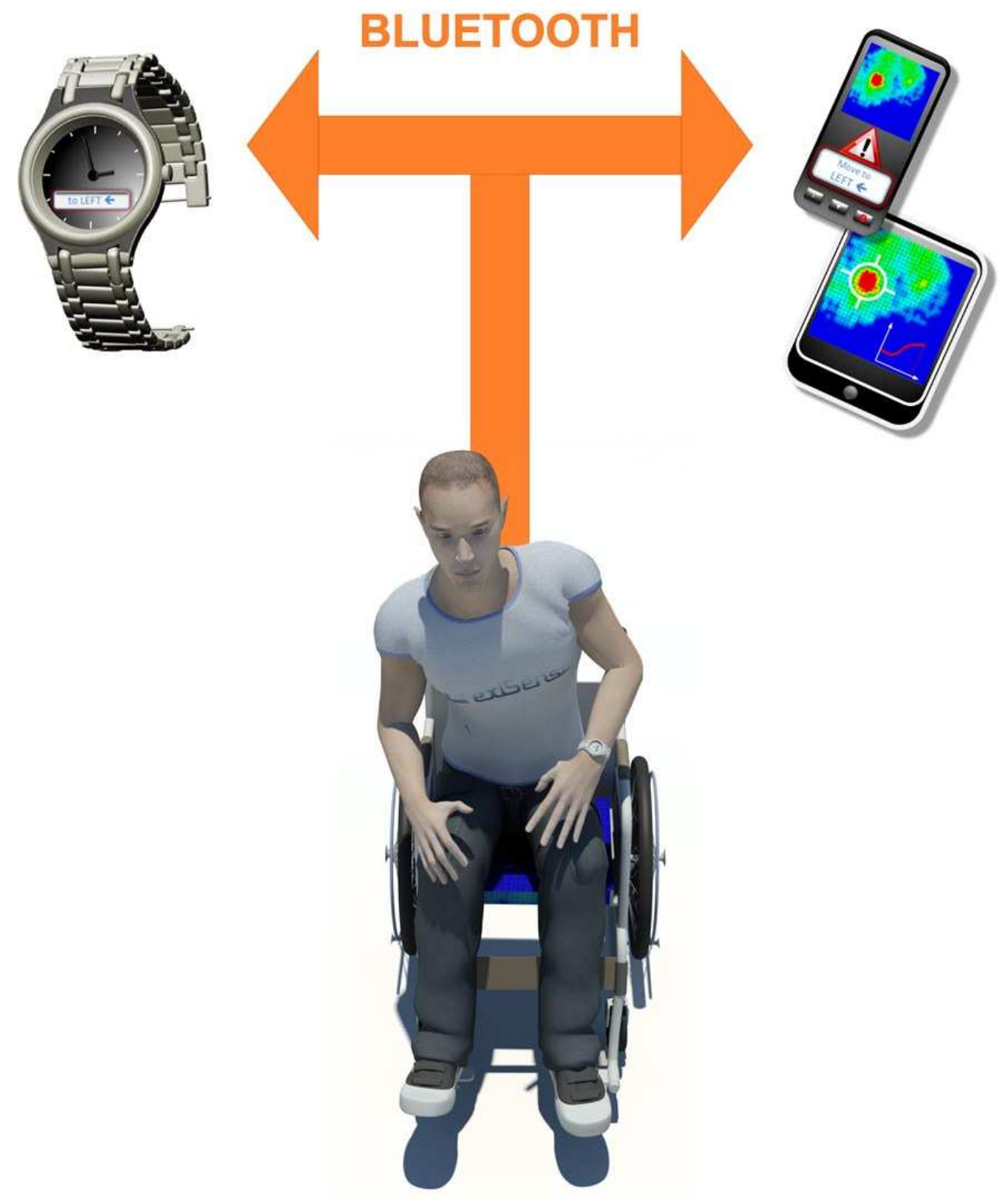

Figure 1 
(a)

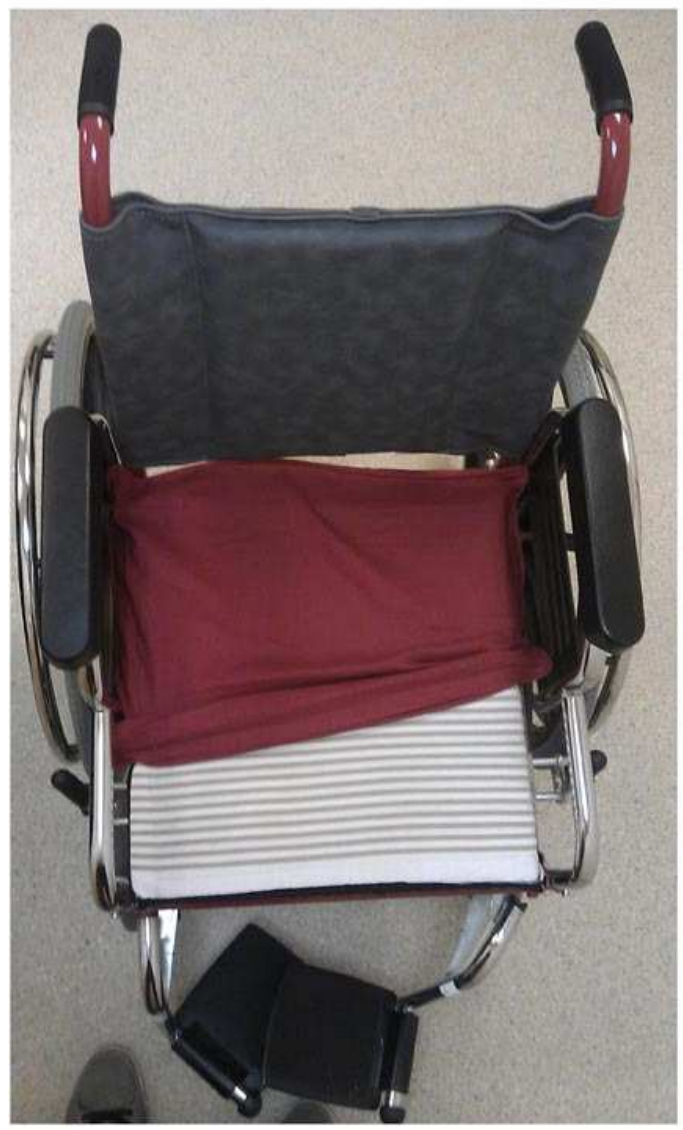

(b)

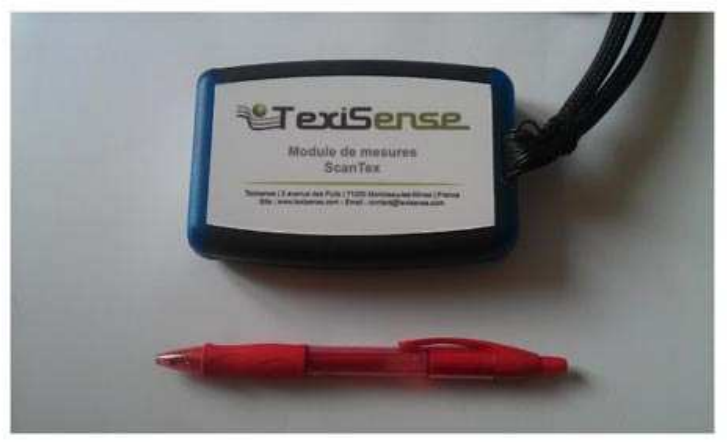

(c)

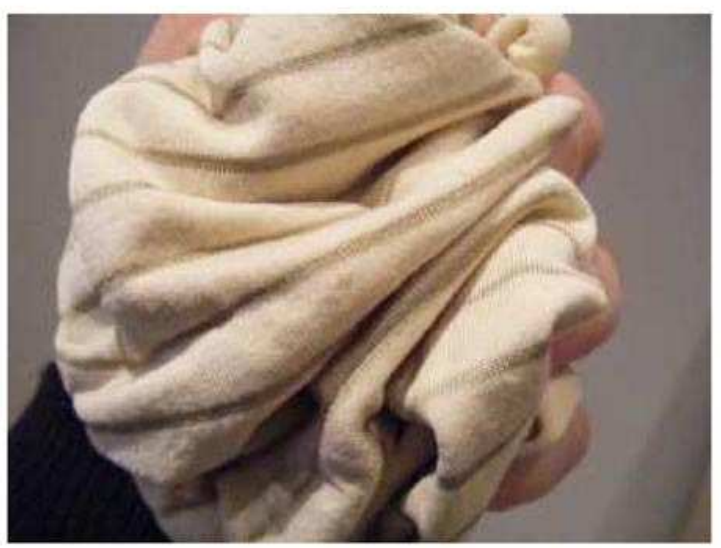

Figure 2 


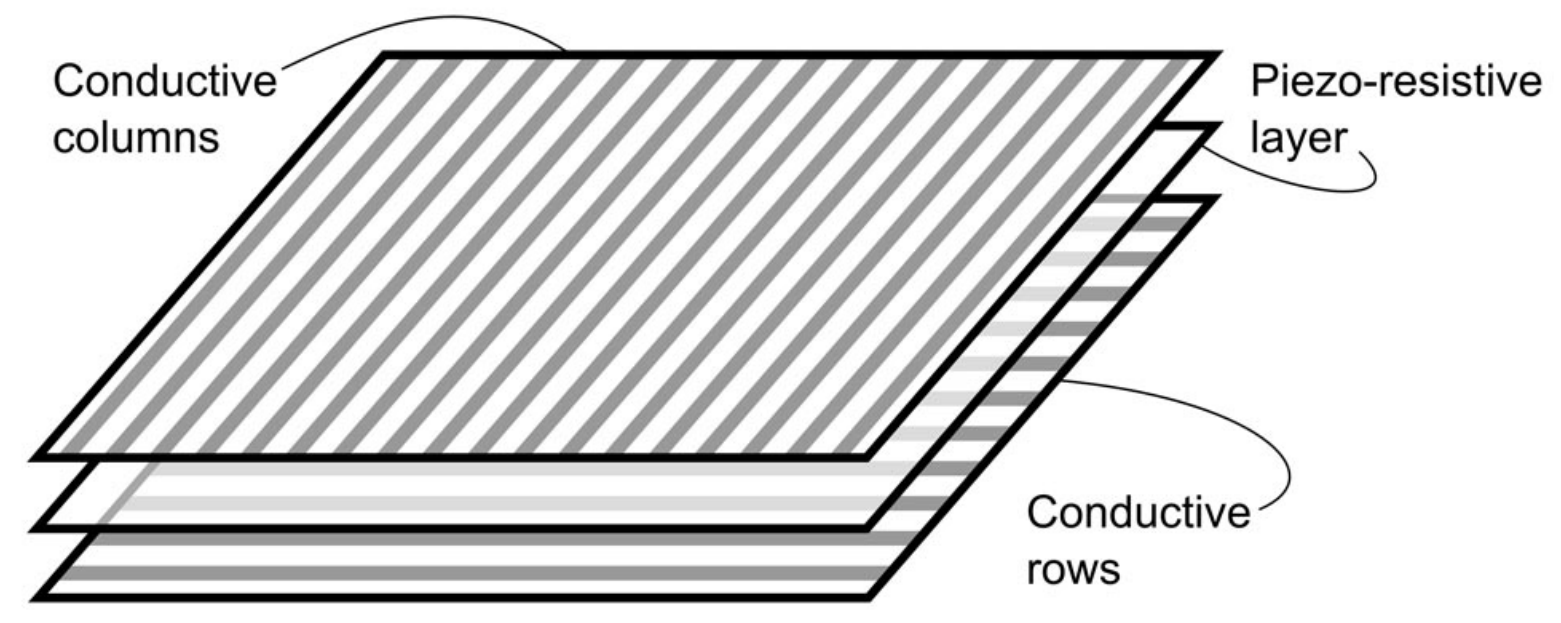

Figure 3 
(a)

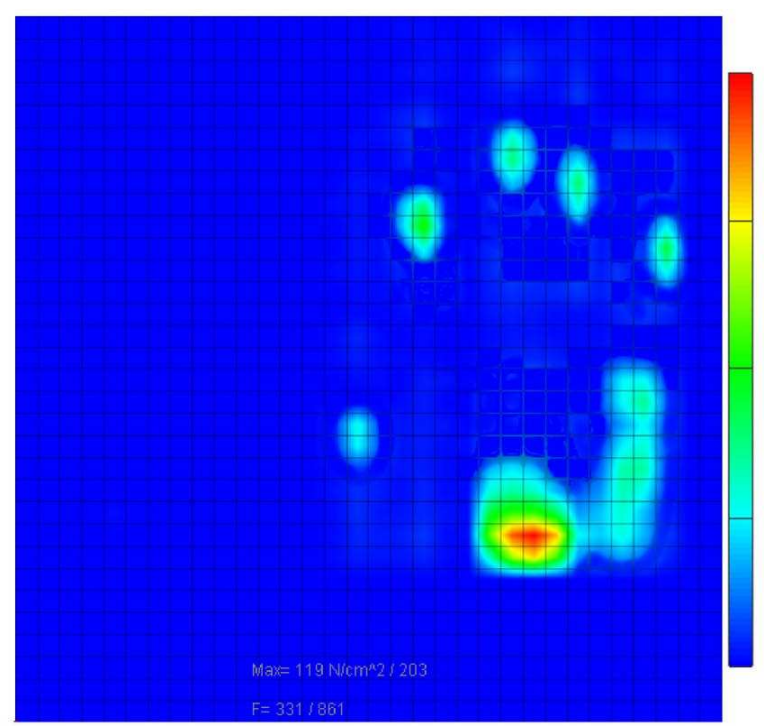

(b)

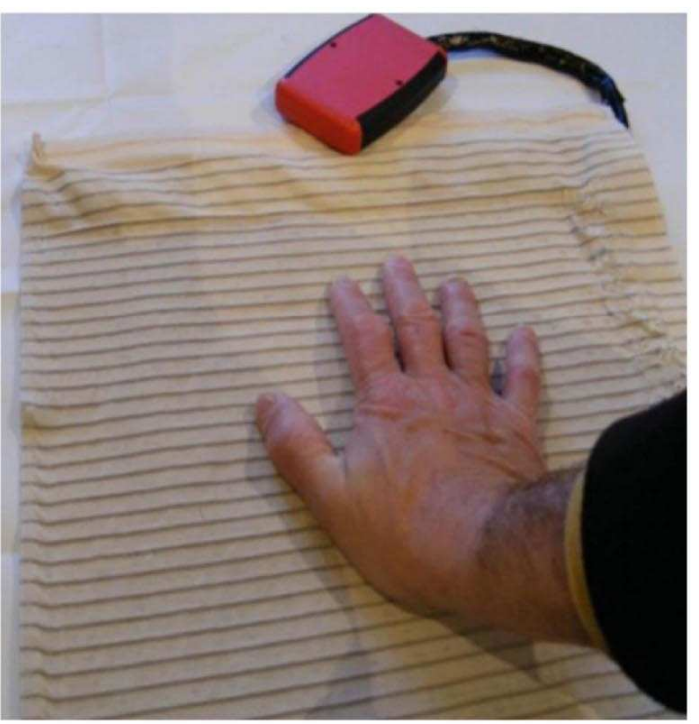

Figure 4 
(a)

(b)
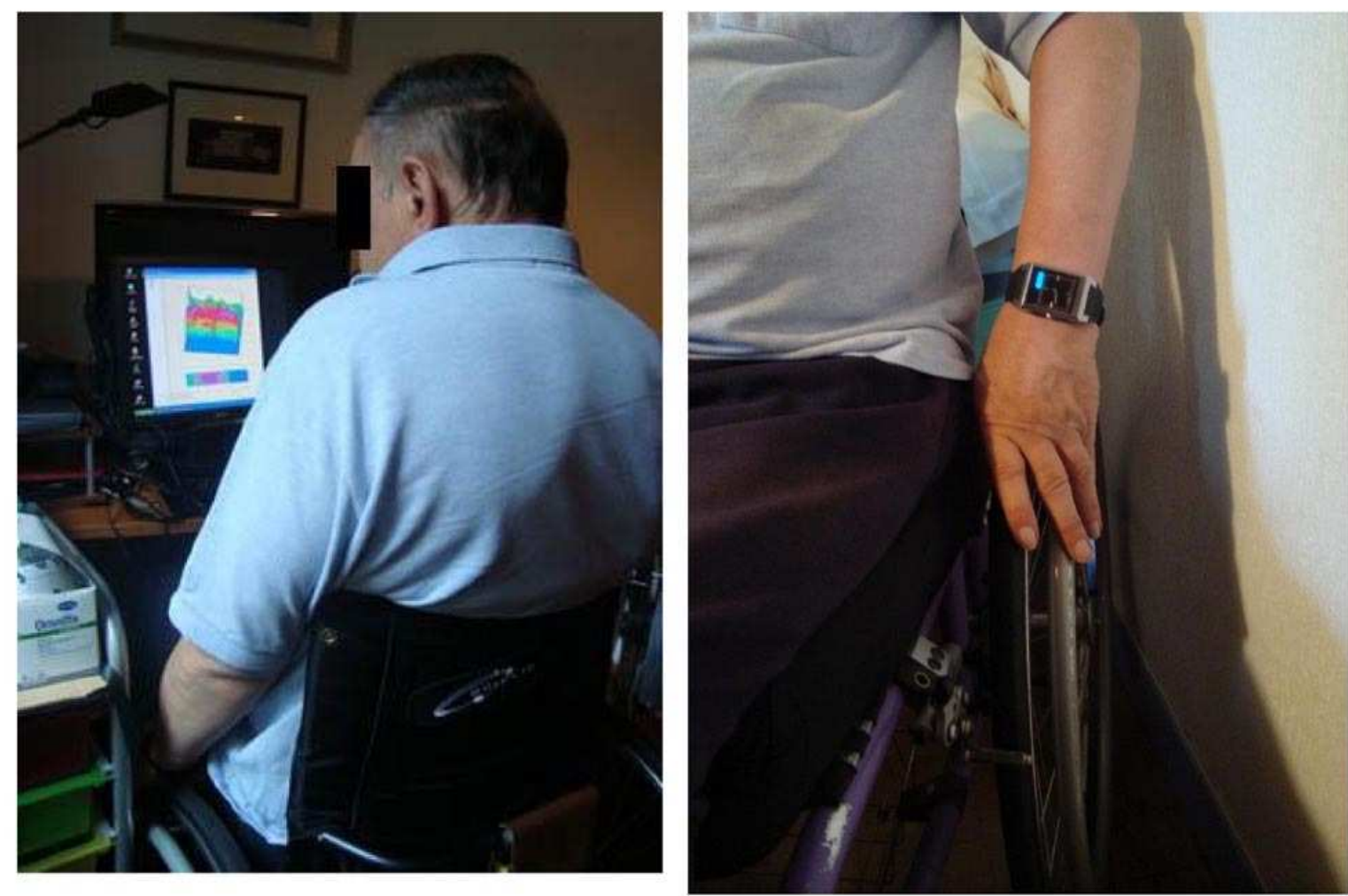

Figure 5 


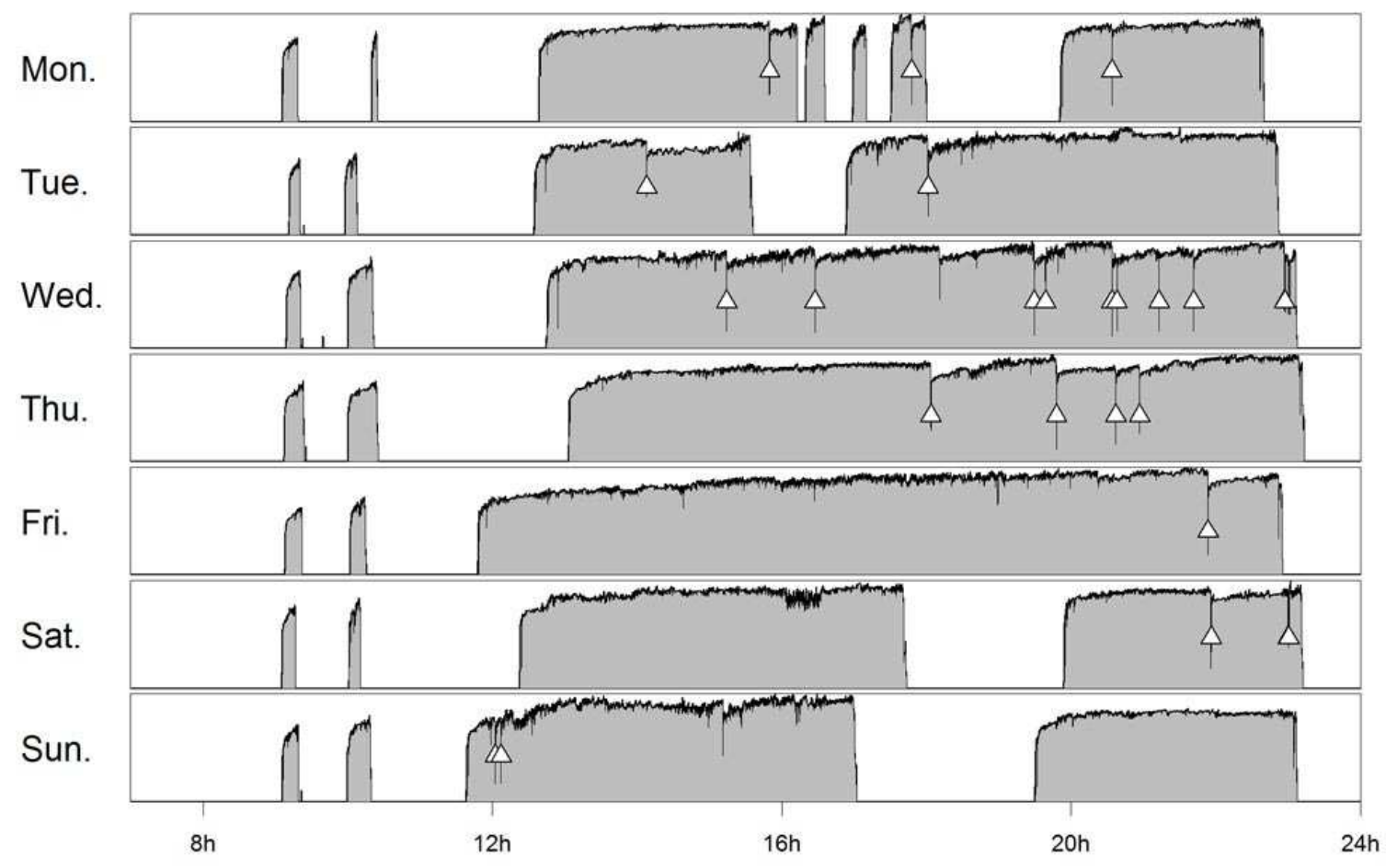

Figure 6 


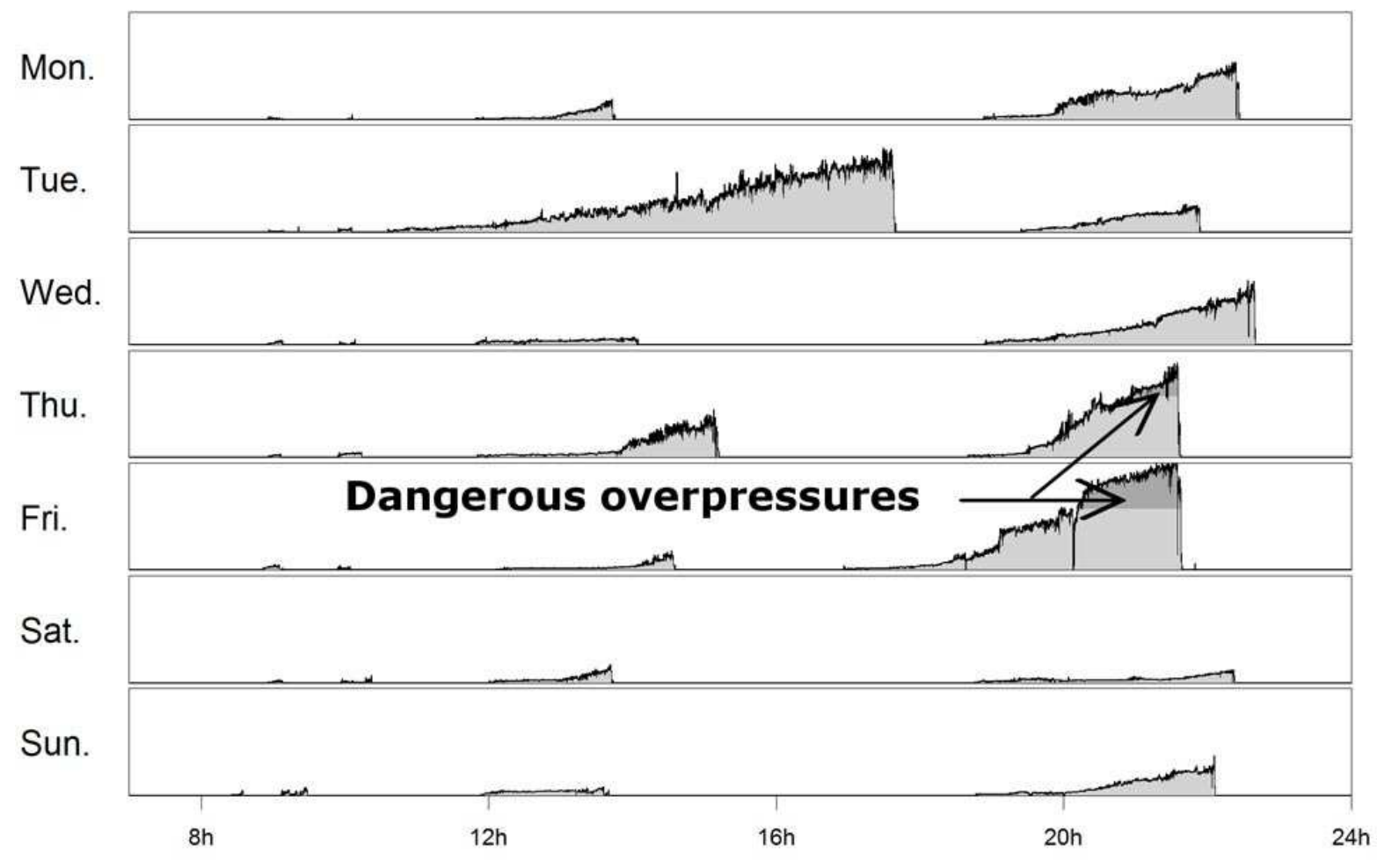

Figure 7 


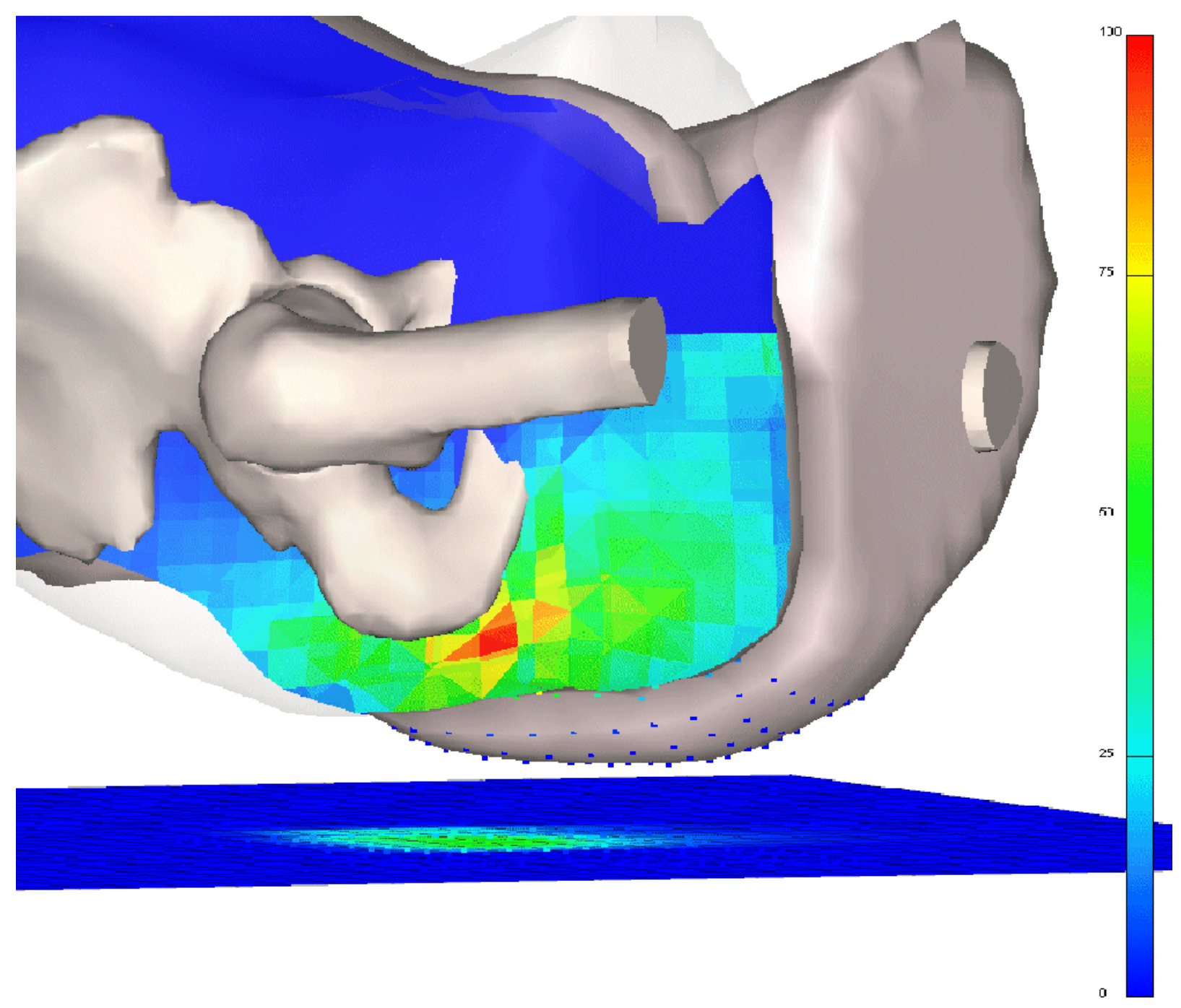

Figure 8 


\section{(a)}

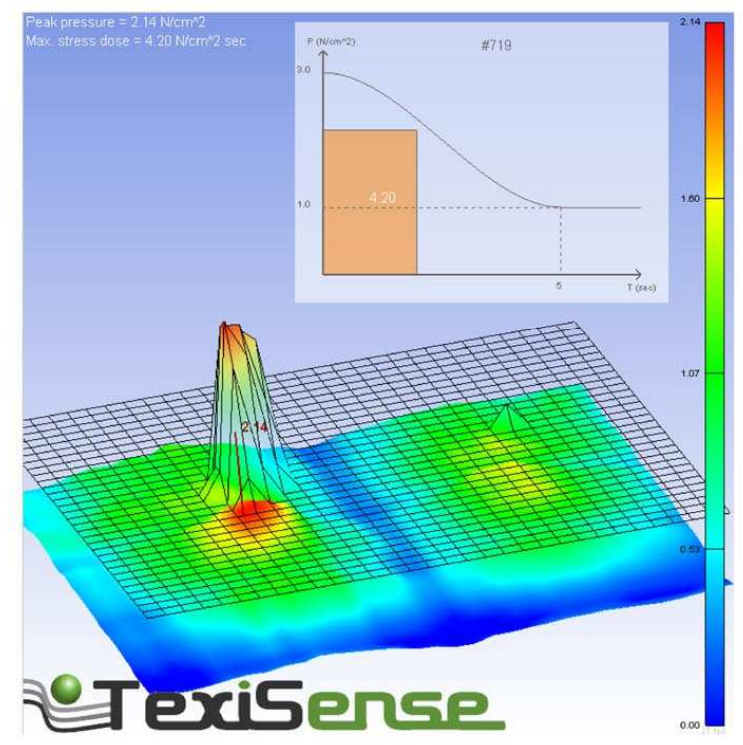

(b)

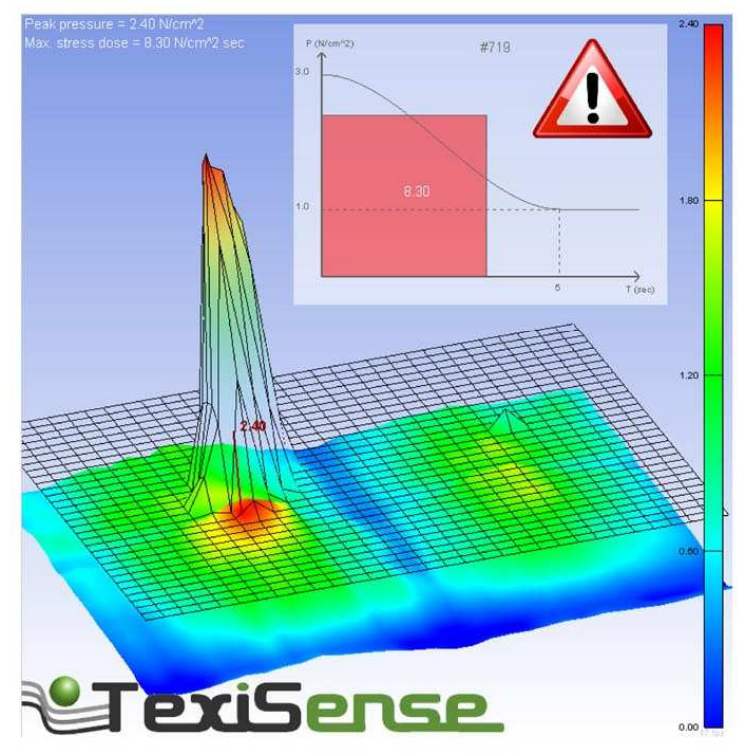

Figure 9 